\title{
Unsaturated Selenacrown Ethers: Synthesis, Structure, and Formation of Silver Complexes
}

Toshio Shimizu, Mari Kawaguchi, Takahiro Tsuchiya, Kazunori Hirabayashi,
and Nobumasa Kamigata*

\section{Supporting Information}

Optimized Structures of Unsaturated Selenacrown Ethers

Pages 2 - 8

X-ray Crystallographic Analysis for Compounds 4,5,8,9,13, and 15

See separate CIF files 
Optimized Structures of Unsaturated Selenacrown Ethers 1,4-Diselenin

Standard orientation:

\begin{tabular}{|c|c|c|c|c|c|c|}
\hline Center & & & Atomic & & Coordinates & (Angstroms) \\
\hline umber & & nber & Type & $X$ & $\mathrm{Y}$ & Z \\
\hline $\begin{array}{l}1 \\
2 \\
3 \\
4 \\
5 \\
6 \\
7 \\
8 \\
9 \\
10\end{array}$ & $\begin{array}{l}34 \\
34 \\
6 \\
6 \\
6 \\
6 \\
1 \\
1 \\
1 \\
1\end{array}$ & $\begin{array}{l}0 \\
0 \\
0 \\
0 \\
0 \\
0 \\
0 \\
0 \\
0 \\
0\end{array}$ & $\begin{array}{c}1.786 \\
-1.786 \\
.6841 \\
.68412 \\
-.6841 \\
-.6841 \\
1.2488 \\
1.2488 \\
-1.2488 \\
-1.248\end{array}$ & $\begin{array}{l}46 \\
46 \\
3 \\
4 \\
4 \\
3 \\
48 \\
48 \\
48 \\
347\end{array}$ & $\begin{array}{r}.000000 \\
.000000 \\
1.464714 \\
-1.464714 \\
1.464715 \\
-1.464714 \\
2.280724 \\
-2.280722 \\
2.280724 \\
-2.280722\end{array}$ & $\begin{array}{l}-.226639 \\
-.226640 \\
.483778 \\
.483778 \\
.483779 \\
.483779 \\
.950202 \\
.950204 \\
.950204 \\
.950206\end{array}$ \\
\hline
\end{tabular}

9-US-3

Standard orientation:

\begin{tabular}{|c|c|c|c|c|c|}
\hline \multirow{2}{*}{$\begin{array}{l}\text { Center } \\
\text { Number }\end{array}$} & \multirow{2}{*}{\multicolumn{2}{|c|}{$\begin{array}{l}\text { Atomic } \\
\text { Number }\end{array}$}} & \multirow{2}{*}{$\begin{array}{c}\text { Atomic } \\
\text { Type }\end{array}$} & \multicolumn{2}{|c|}{$\underset{X}{\text { Coordinates (Angstroms) }}$} \\
\hline & & & & & \\
\hline $\begin{array}{l}1 \\
2 \\
3 \\
4 \\
5 \\
6 \\
7 \\
8 \\
9 \\
10 \\
11 \\
12 \\
13 \\
14 \\
15\end{array}$ & $\begin{array}{l}34 \\
34 \\
34 \\
6 \\
6 \\
6 \\
6 \\
6 \\
6 \\
1 \\
1 \\
1 \\
1 \\
1 \\
1\end{array}$ & $\begin{array}{l}0 \\
0 \\
0 \\
0 \\
0 \\
0 \\
0 \\
0 \\
0 \\
0 \\
0 \\
0 \\
0 \\
0 \\
0\end{array}$ & $\begin{array}{r}.934768 \\
.936462 \\
-2.439815 \\
2.454967 \\
2.455315 \\
-.014331 \\
-.014054 \\
-1.216867 \\
-1.217464 \\
.505781 \\
3.425357 \\
3.426068 \\
-505423 \\
-1.607437 \\
-1.608672\end{array}$ & $\begin{array}{r}1.913808 \\
-1.913264 \\
-.000394 \\
.675527 \\
-.673409 \\
1.292968 \\
-1.293549 \\
.645491 \\
-.647648 \\
1.533787 \\
1.175741 \\
-1.172903 \\
-1.534831 \\
. .414206 \\
2-.417365\end{array}$ & $\begin{array}{c}-.295024 \\
.294751 \\
.000145 \\
-.134834 \\
.134809 \\
1.312355 \\
-1.312173 \\
1.393151 \\
-1.392702 \\
2.248238 \\
-.255237 \\
.255559 \\
-2.248286 \\
2.393608 \\
-2.393156\end{array}$ \\
\hline
\end{tabular}


12-US-4

Standard orientation:

\begin{tabular}{|c|c|c|c|c|c|}
\hline & & & & & \\
\hline & & & & & \\
\hline $\begin{array}{l}1 \\
2 \\
3 \\
4 \\
5 \\
6 \\
7 \\
8 \\
9 \\
10 \\
11 \\
12 \\
13 \\
14 \\
15 \\
16 \\
17 \\
18 \\
19\end{array}$ & $\begin{array}{c}34 \\
34 \\
34 \\
34 \\
6 \\
6 \\
6 \\
6 \\
6 \\
6 \\
6 \\
6 \\
1\end{array}$ & $\begin{array}{l}0 \\
0 \\
0 \\
0 \\
0 \\
0 \\
0 \\
0 \\
0 \\
0 \\
0 \\
0 \\
0 \\
0 \\
0 \\
0 \\
0 \\
0 \\
0 \\
0\end{array}$ & $\begin{array}{r}2.9 \\
-.43 \\
-43 \\
-2.9 \\
1.61 \\
.353 \\
3.20 \\
-2.28 \\
2.28 \\
-3.2 \\
-.35 \\
-1.6 \\
1.95 \\
-.31 \\
4.2 \\
-2.5 \\
2.5 \\
-4.2 \\
-31 \\
-1.9\end{array}$ & $\begin{array}{rr}1 & -.7 \\
3 & -1.9 \\
1 & 1.9 \\
2 & -1.0 \\
-1.55 & 1.1 \\
& -2.1 \\
6 & 2.1 \\
6 & -1 . \\
9 & 1.5 \\
9 & 1.0 \\
4 & -.9 \\
6 & -1.7 \\
3 & 1.4 \\
8 & -3 . \\
2 & 3.1 \\
4 & -1.2 \\
3 & 1.7 \\
8 & .9\end{array}$ & $\begin{array}{r}-.0 \\
1.3 \\
1.1 \\
.2 \\
.0 \\
.0 \\
.2 \\
1.1 \\
2.3 \\
1.5 \\
.5 \\
.2 \\
.5 \\
1.9 \\
2.3\end{array}$ \\
\hline
\end{tabular}


15-US-5

Standard orientation:

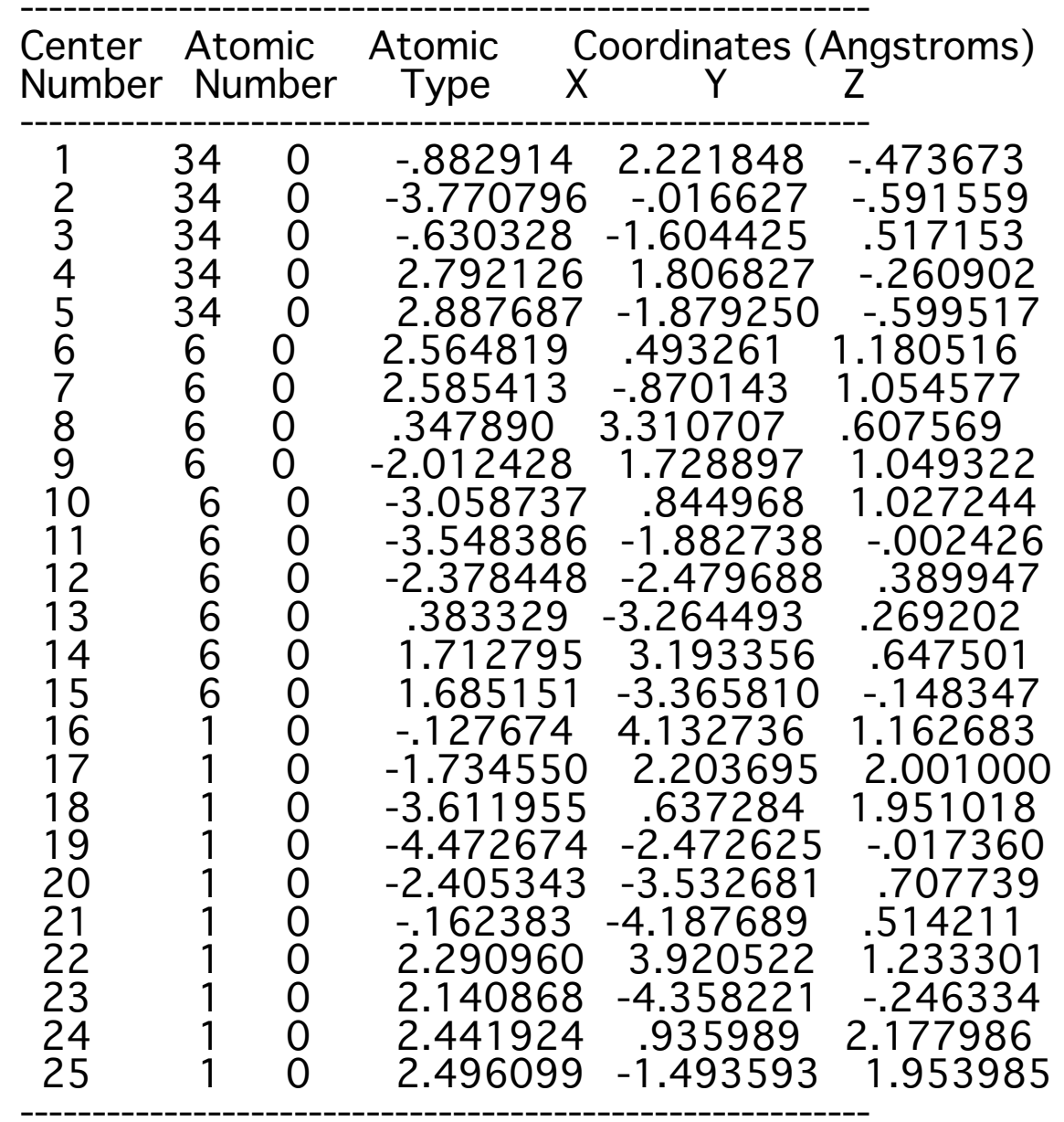


18-US-6

Standard orientation:

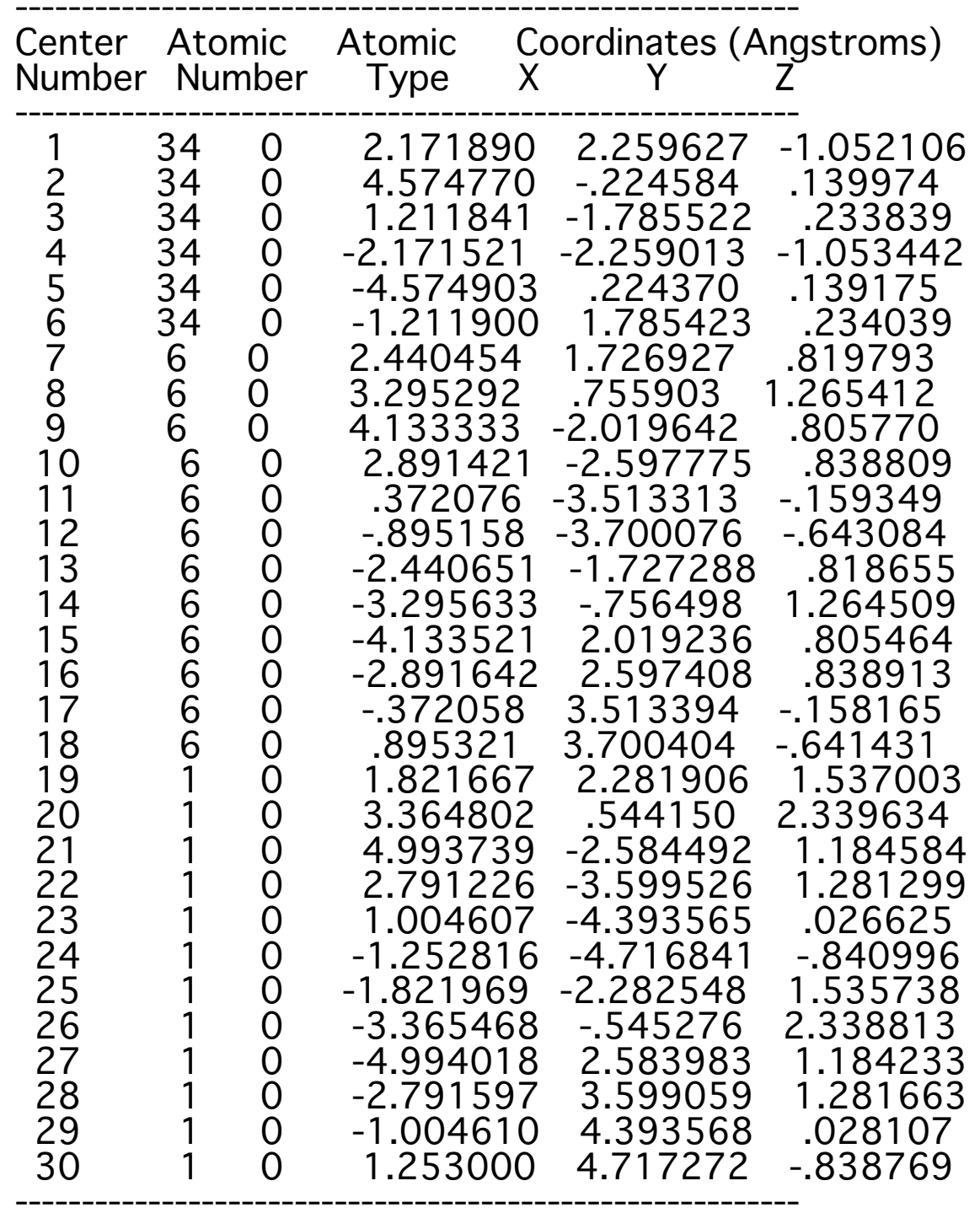


Standard orientation:

\begin{tabular}{|c|c|c|c|c|c|}
\hline $\begin{array}{l}\text { Center } \\
\text { Number }\end{array}$ & Ator & $\begin{array}{l}\text { nic } \\
\text { mber }\end{array}$ & $\begin{array}{r}\text { Atomic } \\
\text { Type }\end{array}$ & ${ }_{X}^{\text {Coordinates }}{ }_{Y}(A$ & $\begin{array}{l}\text { Angstroms) } \\
Z \text { S }\end{array}$ \\
\hline $\begin{array}{l}1 \\
2 \\
3 \\
4 \\
5 \\
6 \\
7 \\
8 \\
9 \\
10 \\
11 \\
12 \\
13 \\
14 \\
15 \\
16 \\
17 \\
18 \\
19 \\
20 \\
21 \\
22 \\
23 \\
24 \\
25 \\
26 \\
27 \\
28 \\
29 \\
30 \\
31 \\
32 \\
33 \\
34 \\
35\end{array}$ & $\begin{array}{l}34 \\
34 \\
34 \\
34 \\
34 \\
34 \\
34 \\
6 \\
6 \\
6 \\
6 \\
6 \\
6 \\
6 \\
6 \\
6 \\
6 \\
6 \\
6 \\
6 \\
6 \\
1 \\
1 \\
1 \\
1 \\
1 \\
1 \\
1 \\
1 \\
1 \\
1 \\
1 \\
1 \\
1\end{array}$ & $\begin{array}{l}0 \\
0 \\
0 \\
0 \\
0 \\
0 \\
0 \\
0 \\
0 \\
0 \\
0 \\
0 \\
0 \\
0 \\
0 \\
0 \\
0 \\
0 \\
0 \\
0 \\
0 \\
0 \\
0 \\
0 \\
0 \\
0 \\
0 \\
0 \\
0 \\
0 \\
0 \\
0 \\
0 \\
0 \\
0\end{array}$ & $\begin{array}{r}-3.508292 \\
-4.993099 \\
-1.844712 \\
1.844523 \\
4.992142 \\
3.509316 \\
.000310 \\
-4.442431 \\
-4.978526 \\
-4.371699 \\
-3.214271 \\
-.685306 \\
.684811 \\
3.213064 \\
4.370157 \\
4.978672 \\
4.443278 \\
2.773486 \\
1.440353 \\
-1.439688 \\
-2.772733 \\
-4.532454 \\
-5.494181 \\
-5.037060 \\
-2.990923 \\
-1.209330 \\
1.208590 \\
2.989361 \\
5.034889 \\
5.494373 \\
4.533940 \\
3.492469 \\
1.123662 \\
-1.123016 \\
-3.491703\end{array}$ & $\begin{array}{cc}2 & 2.112684 \\
9 & -1.158781 \\
2 & -1.810954 \\
3 & -1.810915 \\
2 & -1.159357 \\
6 & 2.112657 \\
& 2.397683 \\
1 & 1.011467 \\
5 & -.227998 \\
9 & -2.894060 \\
1 & -3.126102 \\
5 & -3.132948 \\
& -3.132991 \\
4 & -3.126476 \\
7 & -2.894653 \\
2 & -.229415 \\
8 & 1.010203 \\
6 & 3.379408 \\
3 & 3.507403 \\
8 & 3.508620 \\
3 & 3.380780 \\
4 & 1.472776 \\
1 & -.752778 \\
0 & -3.735826 \\
3 & -4.152011 \\
0 & -3.883225 \\
0 & -3.883273 \\
1 & -4.152446 \\
9 & -3.736665 \\
3 & -.754859 \\
0 & 1.470930 \\
9 & 4.060904 \\
2 & 4.279623 \\
6 & 4.281709 \\
3 & 4.063114\end{array}$ & $\begin{array}{c}-.578849 \\
-1.201484 \\
.660043 \\
.660792 \\
-1.202571 \\
-.577716 \\
.282951 \\
.750320 \\
.530154 \\
-.515995 \\
.179550 \\
1.527855 \\
1.528109 \\
.178485 \\
-.517691 \\
.529514 \\
.750535 \\
.736324 \\
1.018708 \\
1.016922 \\
.734039 \\
1.743043 \\
1.343616 \\
-.740568 \\
. .506255 \\
2.136494 \\
2.136938 \\
.504753 \\
-.743215 \\
1.342521 \\
1.743471 \\
1.207044 \\
1.735079 \\
1.732364 \\
1.203552\end{array}$ \\
\hline
\end{tabular}


24-US-8

Standard orientation:

\begin{tabular}{|c|c|c|c|c|c|}
\hline Cent & & & Atomic & $\mathrm{CoO}$ & Angstrom \\
\hline Number & - $\mathrm{Nu}$ & er & Type & $\mathrm{Y}$ & $Z$ \\
\hline 1 & 34 & & 4.49753 & $5-2.068316$ & .857920 \\
\hline 2 & 34 & 0 & 6.30120 & .918536 & $\begin{array}{l}-.223405 \\
177022\end{array}$ \\
\hline $\begin{array}{l}3 \\
4\end{array}$ & $\begin{array}{l}34 \\
34\end{array}$ & 0 & $\begin{array}{l}2.674323 \\
-983057\end{array}$ & $\begin{array}{l}1.724984 \\
2.272138\end{array}$ & $\begin{array}{l}17703 \\
18876\end{array}$ \\
\hline & $\begin{array}{l}34 \\
34\end{array}$ & 0 & -4.49739 & $4 \quad 2.068375$ & -.857431 \\
\hline$\underline{6}$ & 34 & 0 & .983077 & -2.271835 & -.188470 \\
\hline $\begin{array}{l}7 \\
8\end{array}$ & $\begin{array}{l}34 \\
34\end{array}$ & $\begin{array}{l}0 \\
0\end{array}$ & $\begin{array}{l}-6.3 \\
-2.6\end{array}$ & $\begin{array}{cc}4 & -.918717 \\
8 & -1.724996\end{array}$ & $\begin{array}{l}.222952 \\
-.177388\end{array}$ \\
\hline & & 0 & 4.48 & & -.957417 \\
\hline 10 & 6 & 0 & $\begin{array}{r}1.7 \\
-3.5\end{array}$ & $\begin{array}{l}3.121 \\
3714\end{array}$ & $\begin{array}{r}1.163577 \\
-364845\end{array}$ \\
\hline $\begin{array}{l}11 \\
12\end{array}$ & $\begin{array}{l}6 \\
6\end{array}$ & $\begin{array}{l}0 \\
0\end{array}$ & $\begin{array}{r}-3.53614 \\
.353678\end{array}$ & $\begin{array}{l}3.114 \\
3.3151\end{array}$ & $\begin{array}{l}-.364845 \\
1.176463\end{array}$ \\
\hline 13 & 6 & 0 & 5.10829 & 751 & -1.351471 \\
\hline 14 & 6 & $\begin{array}{l}0 \\
0\end{array}$ & $\begin{array}{l}4.1 \\
5.4\end{array}$ & & \\
\hline 16 & 6 & 0 & & & \\
\hline 17 & 6 & 0 & & & .36 \\
\hline 18 & 6 & 0 & 2.2 & & -.0326 \\
\hline $\begin{array}{ll}19 \\
20\end{array}$ & 6 & $\begin{array}{l}0 \\
0\end{array}$ & $\begin{array}{l}-4.4 \\
-3\end{array}$ & $\begin{array}{lr}3 & 1.3 \\
3 & -3.3\end{array}$ & $\begin{array}{r}.95 \\
-1.17\end{array}$ \\
\hline & 6 & 0 & -5.1 & $3^{-5.0}$ & 1.35 \\
\hline & 6 & 0 & -4.1 & $\begin{array}{l}502 \\
108\end{array}$ & .38 \\
\hline 23 & 6 & 0 & -1. & $\begin{array}{l}-2 . \\
-3\end{array}$ & -1.5 \\
\hline & 1 & 0 & 3.9 & $-1 . \dot{c}$ & -1.6 \\
\hline 2 & 1 & 0 & & & \\
\hline & 1 & 0 & -4.1 & & -.4 \\
\hline & 1 & 0 & -.0 & 4.0 & $\begin{array}{r}1.8 \\
-2.36\end{array}$ \\
\hline & 1 & 0 & $\begin{array}{l}5.0 \\
37\end{array}$ & & $\begin{array}{r}-2.3 \\
-6\end{array}$ \\
\hline & 1 & 0 & & & $\begin{array}{l}-.66 \\
-.86\end{array}$ \\
\hline & 1 & 0 & -1.7 . & & .27 \\
\hline & 1 & 0 & & & \\
\hline & & & & & \\
\hline & 1 & 0 & -3.9 & & 1.6 \\
\hline & & 0 & & & -1.8 \\
\hline & & U & & & \\
\hline & & 0 & & & \\
\hline & 1 & 0 & & -3 & .8 \\
\hline & & & & -3.7 & -1.82 \\
\hline
\end{tabular}


27-US-9

Standard orientation:

\begin{tabular}{|c|c|c|c|c|c|}
\hline $\begin{array}{l}\text { Center } \\
\text { Number }\end{array}$ & & & $\begin{array}{c}\text { Atomic } \\
\text { Type }\end{array}$ & $X_{X}^{\text {Coordinate }}$ & Angstrom \\
\hline $\begin{array}{l}1 \\
2 \\
3 \\
4 \\
5 \\
6 \\
7 \\
8 \\
9 \\
10 \\
11 \\
12 \\
13 \\
14 \\
15 \\
16 \\
17 \\
18 \\
19 \\
20 \\
21 \\
22 \\
23 \\
24 \\
25 \\
26 \\
27 \\
28 \\
29 \\
30 \\
31 \\
32 \\
33 \\
34 \\
35 \\
36 \\
37 \\
38 \\
39 \\
40 \\
41 \\
42 \\
43 \\
44 \\
45\end{array}$ & $\begin{array}{r}34 \\
34 \\
34 \\
34 \\
34 \\
34 \\
34 \\
34 \\
34 \\
6 \\
6 \\
6 \\
6 \\
6 \\
6 \\
6 \\
6 \\
6 \\
6 \\
6 \\
6 \\
6 \\
6 \\
6 \\
6 \\
6 \\
6 \\
1 \\
1 \\
1 \\
1 \\
1 \\
1 \\
1 \\
1\end{array}$ & $\begin{array}{l}0 \\
0 \\
0 \\
0 \\
0 \\
0 \\
0 \\
0 \\
0 \\
0 \\
0 \\
0 \\
0 \\
0 \\
0 \\
0 \\
0 \\
0 \\
0 \\
0 \\
0 \\
0 \\
0 \\
0 \\
0 \\
0 \\
0 \\
0 \\
0 \\
0 \\
0 \\
0 \\
0 \\
0 \\
0 \\
0 \\
0 \\
0 \\
0 \\
0 \\
0 \\
0 \\
0 \\
0 \\
0\end{array}$ & $\begin{array}{r}3.457420 \\
-.035417 \\
-3.359698 \\
-6.190352 \\
-3.772147 \\
-.723148 \\
1.784864 \\
4.420464 \\
5.475839 \\
2.762390 \\
1.442048 \\
-1.430455 \\
-2.679136 \\
-3.681930 \\
-4.697204 \\
-6.585941 \\
-5.701519 \\
-3.694581 \\
-2.551715 \\
-.818424 \\
.116952 \\
2.529096 \\
3.527648 \\
5.103287 \\
5.540181 \\
3.511187 \\
2.748675 \\
3.517295 \\
1.152800 \\
-1.181279 \\
-3.396262 \\
-2.917570 \\
-4.767974 \\
-7.638560 \\
-6.071803 \\
-4.670724 \\
-2.624882 \\
-1.722035 \\
-.053419 \\
2.094898 \\
3.871245 \\
5.167837 \\
5.950097 \\
3.024736 \\
1.656230\end{array}$ & $\begin{array}{lc}0 & 3.636397 \\
& 4.505388 \\
8 & 3.023120 \\
2 & .712028 \\
7 & -2.124748 \\
3 & -3.920856 \\
4 & -2.847070 \\
t & -2.385351 \\
9 & .836242 \\
0 & 4.885701 \\
8 & 5.198481 \\
5 & 4.991145 \\
6 & 4.433136 \\
0 & 1.729942 \\
4 & .813122 \\
1 & -1.186816 \\
9 & -2.233196 \\
1 & -3.896278 \\
5 & -4.573888 \\
1 & -4.195970 \\
-3.804132 \\
6 & -4.249812 \\
8 & -4.085114 \\
7 & -2.084468 \\
1 & -.887880 \\
7 & .943047 \\
5 & 1.999412 \\
5 & 5.348355 \\
0 & 5.905598 \\
9 & 5.827386 \\
2 & 4.822375 \\
0 & 1.722784 \\
4 & 111618 \\
0 & -1.394173 \\
3 & -3.242864 \\
4 & -4.336319 \\
2 & -5.538920 \\
5 & -4.727653 \\
& -4.011519 \\
8 & -5.244115 \\
5 & -4.950041 \\
7 & -2.995144 \\
7 & -.850678 \\
6 & -045253 \\
0 & 1.934329\end{array}$ & $\begin{array}{c}-.881446 \\
-.434237 \\
-.251604 \\
-.043137 \\
-.152602 \\
-.981072 \\
1.476236 \\
-1.025618 \\
.338798 \\
.484033 \\
.657187 \\
.853912 \\
.936293 \\
1.195038 \\
1.230066 \\
.246753 \\
.198451 \\
-.988545 \\
-1.320494 \\
.961952 \\
1.882365 \\
.319712 \\
-.604159 \\
.790741 \\
1.293741 \\
.367517 \\
-.043812 \\
1.130464 \\
1.448257 \\
1.523425 \\
1.669254 \\
1.982690 \\
2.071779 \\
.475309 \\
.426522 \\
-1.238861 \\
-1.835022 \\
1.289956 \\
2.945304 \\
.491451 \\
-1.184003 \\
1.402223 \\
2.310227 \\
.769928 \\
.015839\end{array}$ \\
\hline
\end{tabular}

